\title{
Article \\ Obeticholic Acid Improves Aminotransferases Early during Treatment in Patients with Primary Biliary Cholangitis Not Responding to Ursodeoxycholic Acid: A Study in Clinical Practice
}

\author{
Sara Labanca ${ }^{\dagger}$, Valentina Cacciato ${ }^{\dagger}$, Paolo Borro, Simona Marenco, Giulia Pieri, Antonino Picciotto, \\ Maria Corina Plaz Torres (D) and Edoardo G. Giannini *(D)
}

Citation: Labanca, S.; Cacciato, V.; Borro, P.; Marenco, S.; Pieri, G.; Picciotto, A.; Plaz Torres, M.C.; Giannini, E.G. Obeticholic Acid Improves Aminotransferases Early during Treatment in Patients with Primary Biliary Cholangitis Not Responding to Ursodeoxycholic Acid: A Study in Clinical Practice. Immuno 2021, 1, 457-467. https://doi.org/ 10.3390/immuno1040033

Academic Editor: Stefano Fiorucci

Received: 9 October 2021

Accepted: 9 November 2021

Published: 16 November 2021

Publisher's Note: MDPI stays neutral with regard to jurisdictional claims in published maps and institutional affiliations.

Copyright: (c) 2021 by the authors. Licensee MDPI, Basel, Switzerland This article is an open access article distributed under the terms and conditions of the Creative Commons Attribution (CC BY) license (https:// creativecommons.org/licenses/by/ $4.0 /)$.
Gastroenterology Unit, Department of Internal Medicine, IRCCS Ospedale Policlinico San Martino, University of Genoa, 16132 Genoa, Italy; sara.labanca@hsanmartino.it (S.L.); valentina.cacciato@gmail.com (V.C.); paolo.borro@hsanmartino.it (P.B.); simona.marenco@hsanmartino.it (S.M.); giulia.pieri@hsanmartino.it (G.P.); picciott@unige.it (A.P.); m.plaztorres@gmail.com (M.C.P.T.)

* Correspondence: egiannini@unige.it; Tel.: +39-010-353-7950; Fax:+39-010-353-8638

+ These authors contributed equally to this work.

Abstract: Obeticholic acid (OCA) improves cholestasis and is generally well tolerated in patients with primary biliary cholangitis (PBC) not responding, or intolerant, to ursodeoxycholic acid (UDCA). As $\mathrm{PBC}$ is mainly a cholestatic disorder, less attention is paid to aminotransferase behavior in the course of treatment. In this study we evaluated, in clinical practice, the efficacy of OCA treatment on both alkaline phosphatase (ALP) and alanine aminotransferase (ALT) using updated healthy ranges for aminotransferases. Fifteen PBC patients, non-responders to UDCA, were evaluated at baseline and during OCA treatment with serial measurement of cholestasis indexes and ALT, that were also assessed using updated normal ranges ( $<30 \mathrm{IU} / \mathrm{L}$ in males, $<19 \mathrm{IU} / \mathrm{L}$ in females). Median ALP and ALT decreased from 2.16 to $1.27 \times$ upper limit of normal $(p=0.003)$ and from 0.93 to $0.78 \times$ upper limit of normal $(p=0.008)$, respectively, in the course of OCA treatment. At treatment day-15, median ALT decreased by $29.7 \%$ and ALP by $8.8 \%$. Bilirubin and albumin were unmodified throughout treatment. Using updated normal ranges, ALT levels were normal in $6.7 \%$ of patients at baseline and in $33.3 \%$ of patients at 18 months of treatment. OCA treatment improves cholestasis and, also, indexes of hepatocyte necrosis, with a decline in necro-inflammatory activity even predating the improvement in cholestasis. Use of recalibrated healthy ranges for aminotransferases might be a useful tool to assess hepatic histological activity and its improvement with OCA treatment.

Keywords: treatment; outcome; alanine aminotransferase; alkaline phosphatase; real-life

\section{Introduction}

Primary biliary cholangitis (PBC) is a rare autoimmune cholestatic liver disease, whose prevalence varies between 1.91 and 40.2 on 100,000 people and whose incidence is between 0.3 and 5.8 on 100,000 cases per year, with highest figures observed in Northern Europe and Northern America [1]. PBC tends to affect middle-aged women, who often have comorbid autoimmune diseases, and its course is generally mild to moderate, with a slow progression to more advanced forms of liver disease in most cases, although it may also evolve to cirrhosis and end-stage liver disease, eventually leading to liver transplantation [1-3].

Prognostic assessment is essential, and adequate risk stratification is critical, for the management of patients with PBC [4,5]. Several models such as the UK-PBC and GLOBE scores have recently been proposed, and validated, to assess the prognosis of PBC patients both at diagnosis and in the course of treatment [6-8]. Indeed, treatment of PBC is based on the chronic administration of ursodeoxycholic acid (UDCA), and improvement or normalization of biochemical indexes of cholestasis is currently considered an end-point of 
treatment, mainly due to the slow progression of disease and therefore to the unfeasibility of using harder end-points such as death and occurrence of liver transplantation, and to the close association between these clinical endpoints and the lack of biochemical response to treatment $[9,10]$. If UDCA treatment is started during the early stages of the disease the drug is able to ensure a life expectancy similar to the general population $[1,2,4,5]$. However, after one year of UDCA therapy, up to $40 \%$ of patients may not respond to treatment, according to international response criteria which take into account biochemical parameters-alkaline phosphatase (ALP) and total bilirubin $[9,10]$. In patients who do not obtain a response to UDCA, the administration of obeticholic acid (OCA) has been shown to successfully decrease cholestasis indexes over one year of treatment, and to be generally well tolerated $[10,11]$. Thus, for these patients OCA is the only therapeutic option currently available in clinical practice, and there are published data of real-world effectiveness of OCA replicating those of the pivotal studies, thus enhancing the confidence in the efficacy of the drug outside clinical trials [12,13]. In detail, these studies have shown that OCA is able to determine a decrease in ALP in a proportion of patients similar to the one reported in the registration study, and that OCA-treated patients also show a significant decrease in indexes of liver necro-inflammatory activity such as aminotransferases [12,13].

Indeed, $\mathrm{PBC}$ is mainly a cholestatic disorder and aminotransferases are seldom elevated in these patients-although portal and periportal inflammation are also pathological features of the disease - and less attention is often paid to biochemical indexes of hepatocellular damage behavior in the course of treatment [14]. Monitoring aminotransferases during treatment might provide a more in-depth reflex of hepatic histological activity and of its improvement with therapy, and use of recalibrated healthy ranges for aminotransferases can be a suitable tool to explore the occurrence of these modifications $[15,16]$.

Therefore, in our study we sought to assess the efficacy and tolerability of OCA in a group of patients with PBC not responsive to UDCA who were treated in clinical practice. In particular, we evaluated the efficacy not on the common parameters of cholestasis alone, but also on indexes of hepatic cytolytic activity such as aminotransferases, using updated definition of healthy ranges, and markers of immune activation such as immunoglobulin $\mathrm{M}$.

\section{Patients and Methods}

\subsection{Participants and Follow-Up}

We included in this study, among patients with PBC followed at our Unit, those eligible for a second-line treatment with OCA according to the indications set forth by the Italian Medicines Agency (Agenzia Italiana del Farmaco, AIFA) when the drug was made available in Italy in 2017 [17]. Briefly, OCA was indicated for patients on stable UDCA treatment for at least 12 months and showing an ALP $\geq 1.5$ times the upper limit of normal (ULN), or who were intolerant to UDCA after at least a period of 3 months of treatment [17]. According to these criteria, in the period November 2018-June 2020 we identified 18 patients who were candidate to treatment with OCA, and among them 15 patients accepted OCA treatment, thus representing this study cohort. In these patients the diagnosis of $\mathrm{PBC}$ had previously been made according to serum anti-mitochondrion antibody (AMA) positivity and liver histological findings in 14 patients, and on the basis of AMA positivity in the presence of compatible biochemical and clinical evidence in the remaining patient. Concomitant causes of chronic liver disease such as viral hepatitis infection, alcohol abuse, and non-alcoholic liver disease had been ruled out by means of appropriate clinical and serological work-up, and on the basis of histological findings.

The flow of patients within the study is reported in Figure 1. 


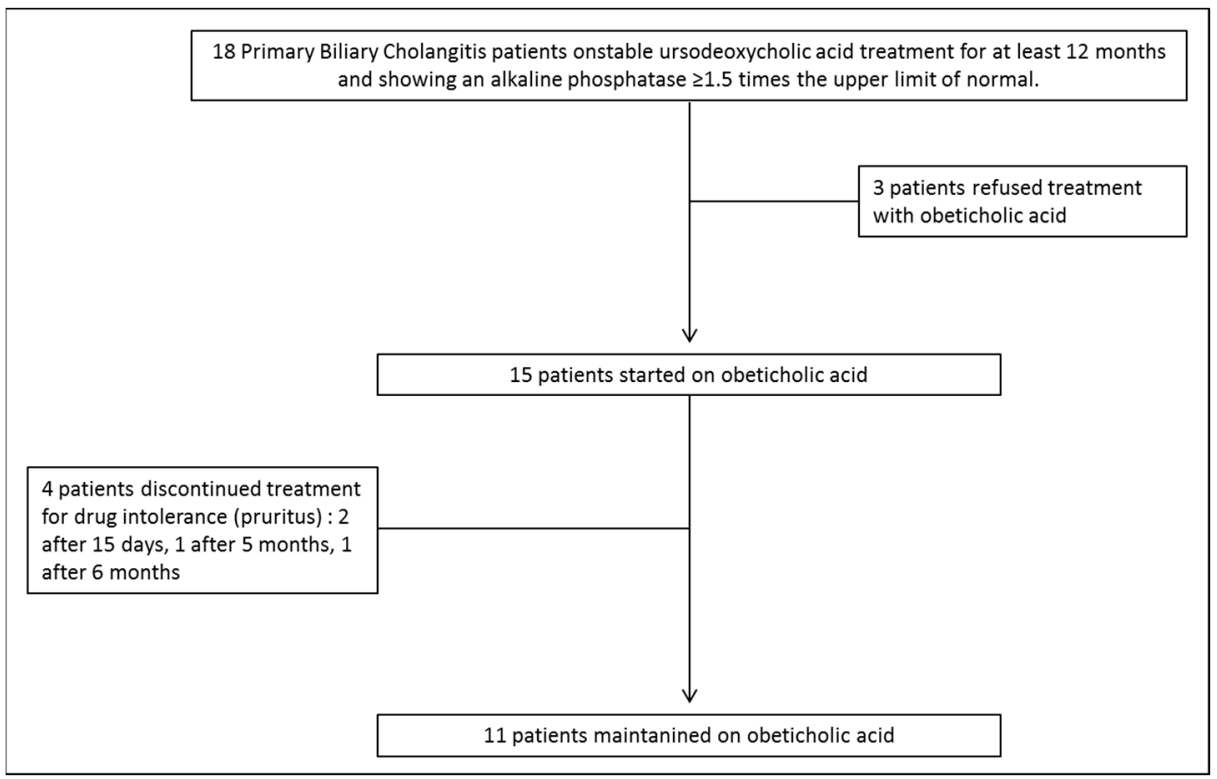

Figure 1. Flow of patients within the study.

Before OCA treatment, all patients underwent biochemical blood and instrumental work-up that provided an initial evaluation of the degree of the disease: aminotransferase, cholestasis indexes, total bilirubin, serum albumin, platelet count, and abdominal ultrasonography with bi-polar measurement of the spleen. Liver stiffness was assessed at baseline in all patients by means of transient elastography, and a stiffness $\geq 16.9 \mathrm{kPa}$ was deemed diagnostic for liver cirrhosis [10]. Historical histological evaluation, when available, was retrieved from records; histological findings were scored according to Ludwig et al. [18].

All patients, in addition to treatment with UDCA ( $15 \mathrm{mg} / \mathrm{kg}$ daily), received OCA at the initial dose of $5 \mathrm{mg}$ daily; the dose was adjusted to 5-10 $\mathrm{mg}$ every other day after one year of treatment, and subsequently increased to $10 \mathrm{mg}$ daily in the case of a partial response to the initial dose, although on the basis of the physician in charge of patients management dose adjustment was adapted to the clinical context on a case-by-case basis.

Serum biochemistry evaluation was repeated after 15 days, 1, 3, 6, 9, 12, 15, and 18 months from the beginning of OCA treatment so as to assess safety and efficacy of the drug. Surveillance for oesophageal varices and for hepatocellular carcinoma were carried out according to guidelines $[19,20]$.

Being this an observational study, the patients included were treated, and followed-up, on the basis of the current standard of care in clinical practice. Drugs were prescribed according to the indications of AIFA, and patients were monitored without any additional test or visit required for the sake of the study alone. Informed consent for data collection and treatment was requested for each patient. According to local rules, the local Ethical Committee was notified of the study, and data regarding study protocol, data acquisition, and copy of the informed consent were provided.

\subsection{Outcomes}

We evaluated changes in serum ALP and aminotransferase levels over the duration of OCA therapy, and the proportion of patients who achieved an ALP level $\leq \mathrm{ULN}$. Furthermore, alanine aminotransferase (ALT) levels were assessed both at baseline and in the course of treatment using updated healthy ranges (i.e., $<30 \mathrm{IU} / \mathrm{L}$ in males and $<19 \mathrm{IU} / \mathrm{L}$ in females) according to Prati et al. [15]. Furthermore, we assessed the course of total bilirubin, serum albumin, and modifications in serum IgM levels. Treatment discontinuation rate and reasons for discontinuation were also recorded. 


\subsection{Statistical Analysis}

Continuous data are reported as median and $95 \%$ confidence interval $(95 \% \mathrm{CI})$ for the median, and categorical data are shown as number and percentage. Data comparison was carried out using the Wilcoxon test for paired samples, while Friedman analysis of variance with internal comparisons was used to assess repeated measurement of ALP levels. Level of significance was set at $p<0.05$ for a two-tailed test.

\section{Results}

\subsection{Baseline Patients Characteristics}

Table 1 shows the baseline demographics and biochemical characteristics of the 15 patients included in this study. Median liver stiffness was $7.3 \mathrm{kPa}(95 \% \mathrm{CI}, 5.3-11.1 \mathrm{kPa})$ and 1 patient $(6.7 \%)$ had a baseline liver stiffness $\geq 16.9 \mathrm{kPa}$. No patient was affected by hepatocellular carcinoma and only $1(6.7 \%)$ presented an overlap PBC-autoimmune hepatitis. Fourteen patients had previously undergone a liver biopsy, a median of 9.5 years before the beginning of OCA treatment $(95 \% \mathrm{CI}, 3.5-21.5$ years), whose results were compatible with stage II in 5 patients (35.7\%), stage III in 7 patients $(50.0 \%)$, and stage IV in 2 patients $(14.3 \%)$.

Table 1. Main clinical and biochemical characteristics of the study population at baseline.

\begin{tabular}{cccc}
\hline Data & Unit & Reference Range & Value \\
\hline Age & years & & $64(56-73)$ \\
Gender & female & & $13(86.7)$ \\
Alanine aminotransferase & $\mathrm{IU} / \mathrm{L}$ & $<40 \mathrm{IU} / \mathrm{L}$ & $37(26-61)$ \\
Alkaline phosphatase & $\mathrm{IU} / \mathrm{L}$ & $<116 \mathrm{IU} / \mathrm{L}$ & $264(193-318)$ \\
Total bilirubin & $\mathrm{mg} / \mathrm{dL}$ & $<1.2 \mathrm{mg} / \mathrm{dL}$ & $0.58(0.44-0.98)$ \\
Albumin & $\mathrm{g} / \mathrm{dL}$ & $>3.5 \mathrm{~g} / \mathrm{dL}$ & $4.1(3.9-4.3)$ \\
INR & ratio & $0.8-1.2$ & $0.99(0.95-1.07)$ \\
Platelet count & $\mathrm{x} 10^{9} / \mathrm{L}$ & $145-450 \times 10^{9} / \mathrm{L}$ & $231(174-277)$ \\
Total cholesterol & $\mathrm{mg} / \mathrm{dL}$ & $<200 \mathrm{mg} / \mathrm{dL}$ & $205(179-217)$ \\
HDL cholesterol & $\mathrm{mg} / \mathrm{dL}$ & $>45 \mathrm{mg} / \mathrm{dL}$ & $64(48-87)$ \\
LDL cholesterol & $\mathrm{mg} / \mathrm{dL}$ & $<130 \mathrm{mg} / \mathrm{dL}$ & $122(100-143)$ \\
Triglycerides & $\mathrm{mg} / \mathrm{dL}$ & $0.4-2.5 \mathrm{~g} / \mathrm{L}$ & $103(67-162)$ \\
Immunoglobulin M & $\mathrm{g} / \mathrm{L}$ & $2.51(2.13-5.80)$ \\
Comorbidities, $\mathrm{n}(\%)$ & $\mathrm{present}$ & & \\
Histological stage, $\mathrm{n}(\%) *$ & $\mathrm{I}$ II, III, IV & & $0,0,5(35.7), 7(50.0), 2(14.3)$ \\
\hline
\end{tabular}

Data are shown as median and $95 \%$ confidence interval for the median or as absolute number and percentage. ${ }^{*}$ Histology was available in 14 patients.

Before starting OCA treatment, all patients were in therapy with UDCA for a median of 108 months (CI95\%, 12-336 months) and their median ALP was $2.16 \times$ ULN while median ALT was $0.93 \times \mathrm{ULN}$, and median bilirubin was $0.48 \times \mathrm{ULN}$.

At baseline, the median estimated probability of liver transplantation-free survival calculated according to the GLOBE score was $96.8 \%, 94.3 \%, 85.3 \%$, and $75.2 \%$ at $3,5,10$, and 15 years, respectively.

\subsection{OCA Treatment and Changes in Biochemical Parameters}

Median treatment duration was 72 weeks (95\%CI, 22-72 weeks), and more in detail 13 patients $(86.7 \%)$ were treated for at least 4 weeks, 12 patients $(80.0 \%)$ for 6 months, 10 patients $(67.0 \%)$ for 12 months, and 9 patients $(60.0 \%)$ for 18 months, including those who dropped out due to intolerance ( 2 patients dropped out after 15 days, one after 5 months, and one after 6 months).

Median ALP decreased from a baseline of $2.16 \times \mathrm{ULN}$ to $1.47 \times \mathrm{ULN}$ at 12-month treatment, and further decreased to $1.27 \times \mathrm{ULN}$ at the end of follow-up, with initial median values of $264 \mathrm{U} / \mathrm{L}$ that decreased to $180 \mathrm{U} / \mathrm{L}$ at 12 months of treatment, and at $155 \mathrm{U} / \mathrm{L}$ at the end of follow-up (Figure 2A, $p=0.003$ ), with a significant decrease beginning at day $15(p=0.005)$ that was maintained at each evaluation time-point in the course of follow- 
up. At the 6-month treatment, the rate of ALP normalization was 9.1\% (1/11 patients), and at 18 months was $22.2 \%$ (2/9 patients). Evaluation of AP behavior after exclusion of patients who normalized AP levels throughout the study showed that the decrease was still statistically significant $(p=0.032)$.
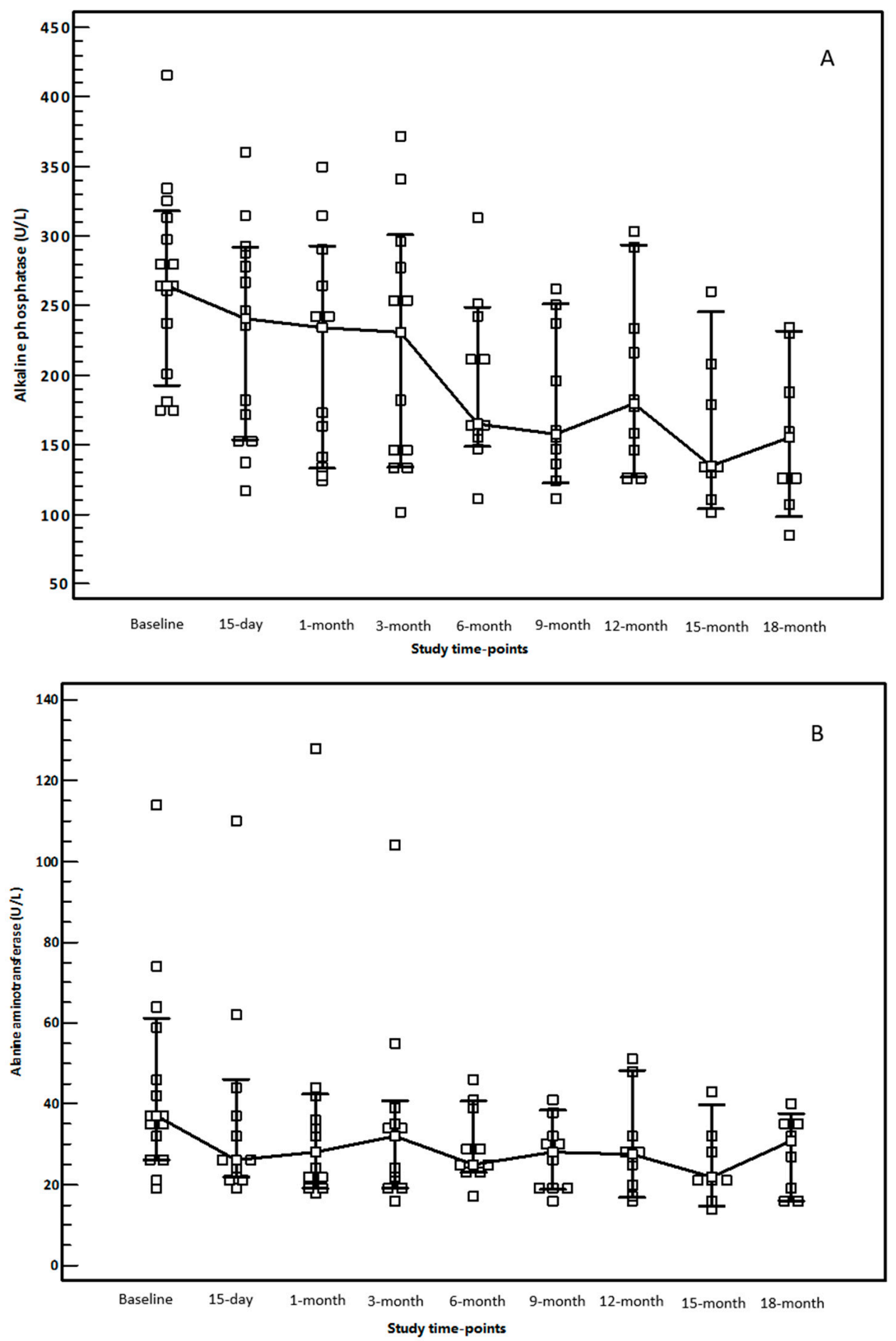

Figure 2. Behavior of median and 95\% confidence interval alkaline phosphatase (A) and alanine aminotransferase $(\mathbf{B})$ values at the various study time-points.

The behavior of median ALT levels throughout the study is shown in Figure 2B: we observed a decrease in median ALT levels that began as early as 15 days after starting OCA treatment (baseline, 37 U/L, 95\%CI: 25-65 U/L; day 15, 26U/L, 95\%CI: 22-46 U/L; 
$p=0.007)$ and was maintained throughout treatment: in particular, ALT levels were $0.93 \times$ ULN before treatment and decreased to $0.78 \times \mathrm{ULN}$ at 18 months $(p=0.008)$.

These results were consistent even after the analyses were repeated excluding the patient with the baseline highest ALT level (i.e., $114 \mathrm{U} / \mathrm{L}$ ).

In the 9 patients who had the longest follow-up we observed a correlation between the decrease in AP and ALT levels that was close to statistical significance $(r=0.617 ; p=0.077)$.

In the 9 patients who had at least 18 -month of follow-up, serum IgM levels significantly decreased from a median baseline value of $2.83 \mathrm{~g} / \mathrm{L}$ to a value of $1.78 \mathrm{~g} / \mathrm{L}$ at the end of follow-up (Figure 3, $p=0.004$ ).

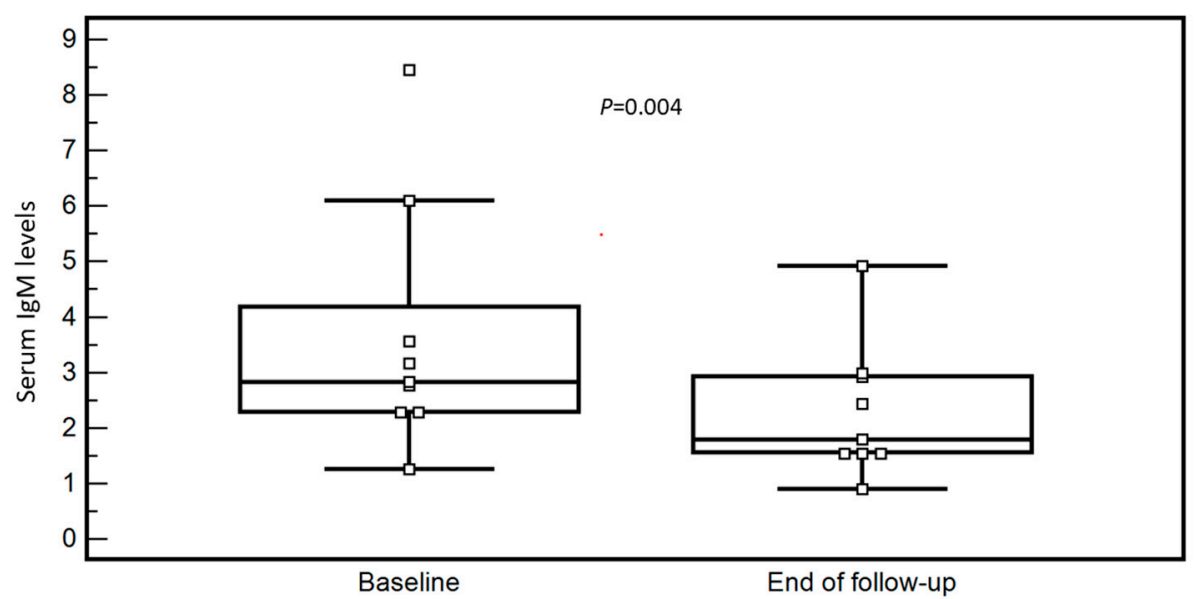

Figure 3. Baseline and end of follow-up median and 95\% confidence interval levels of immunoglobulin M.

Both median serum total bilirubin (baseline, $0.58 \mathrm{mg} / \mathrm{dL}, 95 \% \mathrm{CI}$ : $0.44-0.94 \mathrm{mg} / \mathrm{dL}$; 6-month, $0.38 \mathrm{mg} / \mathrm{dL}, 95 \% \mathrm{CI}: 0.29-0.91 \mathrm{mg} / \mathrm{dL}$; end of follow-up, $0.60 \mathrm{mg} / \mathrm{dL}, 95 \% \mathrm{CI}$ : $0.35-0.71 \mathrm{mg} / \mathrm{dL} ; p=0.641$, Figure 4A) and albumin (baseline, $4.1 \mathrm{~g} / \mathrm{dL}, 95 \% \mathrm{CI}: 3.8-4.3 \mathrm{~g} / \mathrm{dL}$; 6-month, 4.2, 95\%CI: 4.0-4.5 g/dL; end of follow-up, $4.2 \mathrm{~g} / \mathrm{dL}, 95 \% \mathrm{CI}: 4.0-4.4 \mathrm{~g} / \mathrm{dL}$; $p=0.148$, Figure 4 B) were unmodified throughout the study.

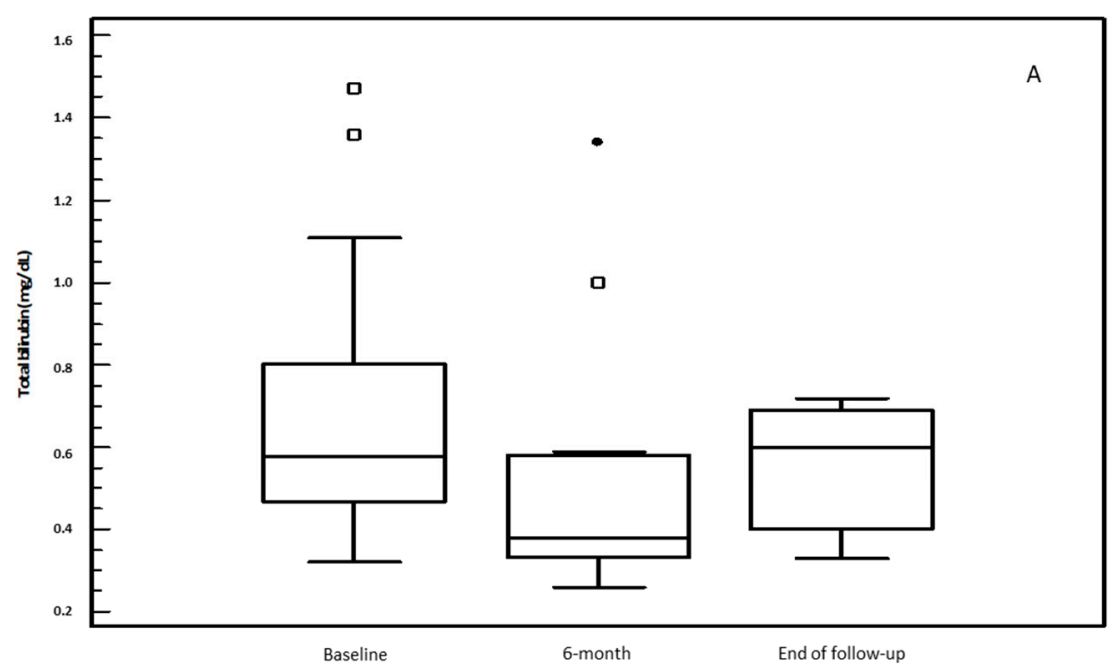

Figure 4. Cont. 


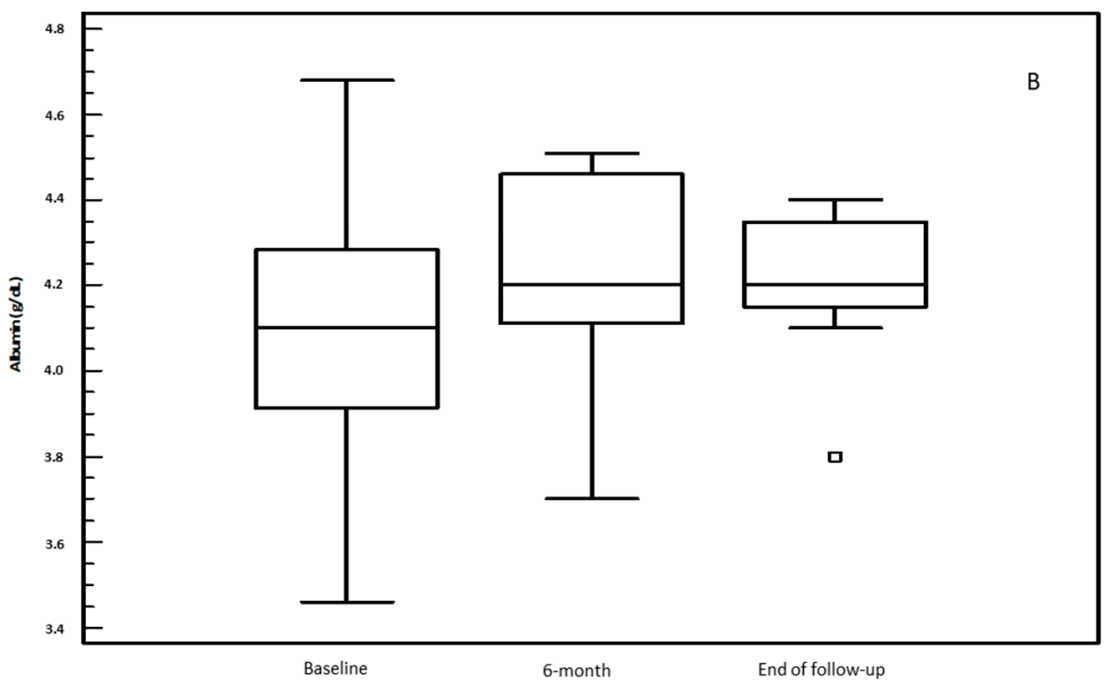

Figure 4. Behavior of serum total bilirubin (A) and albumin (B) in the course of obeticholic acid treatment (median and 95\% confidence interval levels).

Total cholesterol levels (baseline, $205 \mathrm{mg} / \mathrm{dL}, 95 \% \mathrm{CI}$ : 179-217; 12-month, $204 \mathrm{mg} / \mathrm{dL}$, 95\%CI: 187-225; $p=1.00$ ), high-density lipoprotein cholesterol (baseline, $64 \mathrm{mg} / \mathrm{dL}$, 95\%CI: 48-87; 12-month, $74 \mathrm{mg} / \mathrm{dL}, 95 \% \mathrm{CI}$ : 43-94; $p=0.742$ ), and triglycerides (baseline, $103 \mathrm{mg} / \mathrm{dL}$, 95\%CI: 67-162; 12-month, $103 \mathrm{mg} / \mathrm{dL}, 95 \% \mathrm{CI}$ : 55-155, $p=0.547$ ) were unchanged in the course of OCA treatment.

\subsection{Evaluation of Alanine Aminotransferases Modification during OCA Treatment According to Up-Dated Upper Limit of Normal}

Based on the updated upper normal levels of ALT proposed by Prati et al. (i.e., $<19$ U/L in females and $<30 \mathrm{U} / \mathrm{L}$ in males), only $1 / 15$ patient $(6.7 \%)$ had normal ALT at baseline, and this prevalence increased to $2 / 13$ patients $(15.4 \%)$ after 15 days of OCA treatment, to $4 / 13$ patients (30.8\%) after 1 month of OCA, and finally up to $3 / 9$ patients $(33.3 \%)$ at 18 months (Figure 5) [15]. Considering only those patients who had at least 18-month follow-up, these proportions were $11.1 \%$ (1/9 patients) at baseline, $11.1 \%(1 / 9$ patients) at 15-day, $22.2 \%$ (2/9 patients) at 1-month, and 33.3\% (3/9 patients) at 18-month follow-up, while in the 2 remaining patients who continued to be treated but who had a shorter follow-up ALT levels normalized in one male patient at 6 months and continued to decline at the last time-point available (month 9).

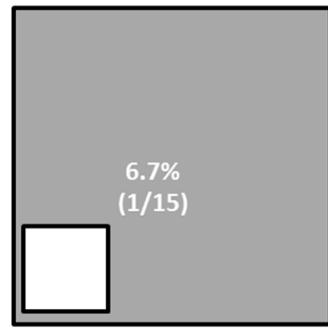

Baseline

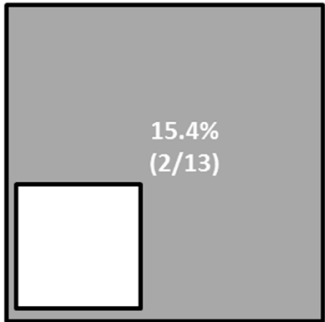

Day 15

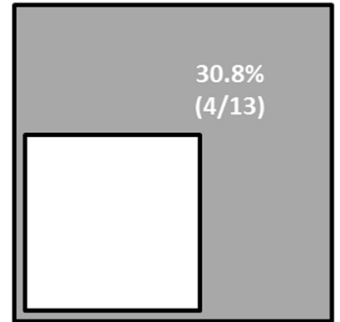

One month

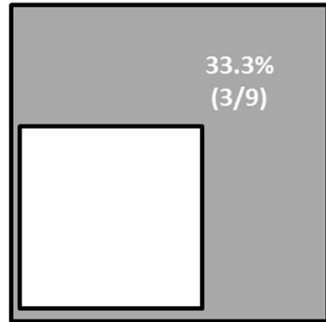

18 months

Figure 5. Proportion of patients with normal alanine aminotransferase (white box) throughout the study. 


\subsection{OCA Tolerability}

As far as OCA dose is concerned, excluding the 4 patients who discontinued the drug due to intolerance, 3 patients continued at the initial dose of $5 \mathrm{mg}$ daily, 4 patients up-titrated to 5-10 mg every other day after 1 year, of which 1 further increased to $10 \mathrm{mg}$ daily after 15 months and 1 at 18 months. Three patients adjusted the dose at 5-10 mg every other day after 9 months, of which 1 further increased to $10 \mathrm{mg}$ daily after 1 year, and lastly 1 patient up-titrated to 5-10 mg every other day at 18 months.

Overall, 4 patients discontinued treatment (26.7\%), 2 after 15 days, one after 5 months and one after 6 months, all for intolerance due to worsening of pruritus. All these patients had complained about pruritus before the initiation of OCA, and their symptoms worsened following the initiation of drug, thus leading to drug withdrawal. Two patients had PBC stage III on historical histology and 2 had stage IV. None of the patients developed portal hypertension-related complications of liver disease (ascites, variceal bleeding) or hepatocellular carcinoma in the course of treatment.

\section{Discussion}

$\mathrm{PBC}$ is a chronic inflammatory liver disease secondary to immune dysregulation that leads to a non-suppurative destruction of small intrahepatic bile ducts and retention of bile acids, with progressive liver fibrosis, and ultimately to the development of advanced liver disease and liver-related death [1,2]. This relatively rare disease often afflicts young and middle-aged patients and its course is life-long due to the absence of a definitive cure, although treatment with UDCA-if initiated at earlier stages of disease-is not only able to improve the cholestatic biochemical picture characterizing PBC, but also to decrease the incidence of liver-related events, and eventually improve patients' survival [21]. Indeed, UDCA is also able to improve transplant-free survival of PBC patients even in those without biochemical improvement [22]. However, a variable proportion of patients, ranging from $12 \%$ to $40 \%$ according to both criteria used to define the outcome and severity of disease, do not respond to UDCA treatment, and in these patients second-line treatment with OCA has shown to be able to improve liver biochemistry [11]. As a fact, one-year, add-on treatment with OCA in UDCA non-responders, or monotherapy in UDCA-intolerant patients, was able to decrease ALP levels and normalize serum bilirubin more frequently as compared to placebo [11]. These positive results were replicated in at least three recent, multicenter, reallife studies performed in Canada, Spain and Portugal, and Italy, although the cut-point of observation in these real-life cohorts was at 12 months as in the registration trial $[12,13,23]$.

In this study we deemed it of interest to assess the outcome of a relatively small group of PBC patients non-responder to UDCA who were treated with OCA in a single center, real-life setting. Patients included in this study were slightly different from those enrolled in the registration trial as the criteria for OCA prescription in Italy include an ALP at least above 1.5 the upper limit of normal after at least 12 months of treatment with UDCA rather than above 1.67 as in the POISE study, and our cohort was older (median age, 65 versus 56 years) and had a longer median duration of disease (12.5 versus $8 / 9$ years), thus highlighting a more difficult to treat subset of patients [11]. Lastly, the median duration of OCA therapy in our study was longer as compared to previous studies, since median on-treatment follow-up was 18 months, thus allowing us to provide an extended picture of on-treatment outcome.

All in all, in our study we observed that OCA treatment determined an improvement in cholestasis that was comparable to the one observed in the registration trial, with a significant, progressive decrease in ALP that began early in the course of OCA administration and that was evident and actual throughout the treatment up to 18 months; moreover, the rate of ALP normalization, bearing in minds the limitation related to the small number of patients included, was similar and even better to the one observed in larger, multicenter, real-life studies [23]. The decrease in cholestasis indexes was accompanied by a decrease in markers of immune activation, such as serum IgM, while from the safety point of view both serum albumin and bilirubin — that were normal in almost all patients at enrolment- 
showed no unexpected deterioration, emphasizing the safety side of the drug. It may be of interest to explore whether these findings are limited to OCA treatment or may also apply to UDCA monotherapy: although this was not a question that could be evaluated in the present study, the availability of large cohorts of patients treated with UDCA alone may help address this issue in the future. Furthermore, we also feel that the observed, significant decrease in IgM serum levels, though examined in the subgroup of patients who had paired data at baseline and at least at 18-month follow-up, merits attention and is worth being explored as a potential useful tool for monitoring disease activity more in detail in future studies and in larger series. Lastly, we observed no significant modification in total cholesterol, high-density lipoprotein cholesterol, and triglycerides in the course of OCA treatment; previous studies on this issue reported inconsistent results, while a recent meta-analysis of three studies reported decreased total and high-density cholesterol levels, and unchanged triglycerides in PBC patients treated with OCA: we feel that also on this topic well-performed studies examining not only serum levels but also lipoprotein composition may help clarify this important clinical issue [24].

As far as biochemical improvement is concerned, of note we observed that median ALT decrease (-29.7\%) was even more pronounced than ALP decrease (-8.8\%) 15 days after the beginning of treatment, and was consistent throughout the course of OCA therapy. Moreover, when we examined ALT according to the updated healthy ranges proposed by Prati et al. we found that only $6.7 \%$ of patients had normal ALT at baseline, and that this proportion progressively increased in the course of treatment up to $33.3 \%$ at the end of follow-up [15]. We feel that this novel finding may be of interest, since PBC is usually considered a liver disease where aminotransferases are normal or only minimally elevated, while using a more stringent criterion we have shown that ALT can be considered elevated in the majority of patients, and that treatment with OCA is able to improve not only cholestasis but also indexes of liver cell damage, and that this improvement in necro-inflammatory activity even predates the improvement in cholestasis. This figure is particularly relevant, as the majority of patients with PBC are females, and the updated upper limit of normal of ALT in females is as low as $19 \mathrm{U} / \mathrm{L}$, thus making the achievement of ALT normalization especially challenging. Lastly, this finding may not be related to the potential overinclusion of patients with PBC-autoimmune hepatitis overlap conditions, as the majority of patients in our study underwent liver biopsy in the course of their disease, and just one patient had such features.

The "normalisation" of aminotransferases, especially using more stringent criteria to define the healthy ranges, might be a debatable end-point, and more so since response to treatment is assessed on decrease and normalization of ALP levels [10]. However, aminotransferases are part of validated prognostic scores to predict patients' outcome, and more recently a secondary analysis of OCA registration study data showed that treatment with OCA is also associated in an improvement in the aspartate aminotransferase-toplatelet ratio index (APRI), besides improvement in GLOBE score, thus emphasizing how (successful) response to treatment may also include the evaluation of hepatic necroinflammatory activity [25].

In our study the tolerability of OCA was lower than reported in the registration study, though we have to bear in mind the differences in patient populations, and indeed the reason for discontinuation was pruritus, that was already present before treatment, while no patient experienced worsening of liver disease or development of portal hypertensionrelated events, a relevant finding as more than half of the population had at least stage III-IV disease according to Ludwig.

Our study undoubtedly has some shortcomings, such as the relatively small cohort, and the fact that drug titration took longer as compared to the registration trial and was adapted to the clinical context, on the basis of the physician in charge of the patient. While the first limitation of this study is inherent to a single-center experience in a rare disease, the latter represents a smoother approach to treatment that might have been attributed to a cautious initial approach to the drug, as also reported in other real-life studies [23]. As 
a fact, it has to be emphasized that OCA should be adequately titrated in patients with limited liver function so as to avoid the occurrence of side effects [26,27]. Furthermore, the updated healthy ranges for ALT that we applied in this study was derived from a population of 6000 healthy blood donors, almost $50 \%$ female, whose median age was approximately 30 years, while our study included patients whose median age was 64 years and with a prevalence of female patients (i.e., $86.7 \%$ ). However, this difference should reinforce our results rather than being a drawback as aging is associated with decreased aminotransferase activity, and we avoided gender-related bias by applying gender-specific upper limit of normal $[15,16]$. Lastly, the results of this study were not biased by other potential confounding factors, as in the course of treatment none of the patients modified dietary habits and physical activity, with consequent absence of variations in body mass index, and none of the patients reported any alcohol use before and during treatment.

To conclude, in this study we found that OCA treatment of PBC patients not responding to UDCA alone is safe and effective in decreasing not only cholestasis indexes, as reported in the registration trial, but even able to significantly improve biochemical indexes of liver necro-inflammatory activity quite early and consistently throughout treatment when aminotransferases are re-assessed with updated healthy ranges. We feel this novel finding is worth being explored in larger cohorts.

Author Contributions: S.L., V.C.: data collection, analysis, and interpretation, writing of the manuscript. P.B., S.M., G.P., A.P. and M.C.P.T.: data collection and analysis. E.G.G.: data analysis and interpretation, writing of the manuscript. All authors have read and agreed to the published version of the manuscript.

Funding: This research received no external funding.

Institutional Review Board Statement: The study was conducted according to the guidelines of the Declaration of Helsinki, and approved by the Ethics Committee of the Regione Liguria. The ID number of the Ethics Committee of the Regione Liguria submission is \#11986, the date is 9 November 2021.

Informed Consent Statement: Informed consent was obtained from all subjects involved in the study.

Conflicts of Interest: S.L., received lecture fees from Intercept; V.L., P.B., S.M., G.P., A.P., M.C.P.T. and E.G.G. have no conflict of interest to declare.

\section{References}

1. Carey, E.J.; Ali, A.H.; Lindor, K.D. Primary biliary cirrhosis. Lancet 2015, 386, 1565-1575. [CrossRef]

2. Imam, M.H.; Lindor, K.D. The natural history of primary biliary cirrhosis. Semin. Liver Dis. 2014, 34, 329-333. [CrossRef]

3. Floreani, A.; Franceschet, I.; Cazzagon, N. Primary biliary cirrhosis: Overlaps with other autoimmune disorders. Semin. Liver Dis. 2014, 34, 352-360.

4. Goel, A.; Kim, W.R. Natural History of Primary Biliary Cholangitis in the Ursodeoxycholic Acid Era: Role of Scoring Systems. Clin. Liver Dis. 2018, 22, 563-578. [CrossRef]

5. Corpechot, C.; Carrat, F.; Poupon, R.; Poupon, R.E. Primary biliary cirrhosis: Incidence and predictive factors of cirrhosis development in ursodiol-treated patients. Gastroenterology 2002, 122, 652-658. [CrossRef]

6. Lammers, W.; Hirschfield, G.; Corpechot, C.; Nevens, F.; Lindor, K.D.; Janssen, H.L.; Floreani, A.; Ponsioen, C.Y.; Mayo, M.J.; Invernizzi, P.; et al. Development and Validation of a Scoring System to Predict Outcomes of Patients with Primary Biliary Cirrhosis Receiving Ursodeoxycholic Acid Therapy. Gastroenterology 2015, 149, 1804-1812. [CrossRef] [PubMed]

7. Carbone, M.; Nardi, A.; Flack, S.; Carpino, G.; Varvaropoulou, N.; Gavrila, C.; Spicer, A.; Badrock, J.; Bernuzzi, F.; Cardinale, V.; et al. Pretreatment prediction of response to ursodeoxycholic acid in primary biliary cholangitis: Development and validation of the UDCA Response Score. Lancet Gastroenterol. Hepatol. 2018, 3, 626-634. [CrossRef]

8. Carbone, M.; Sharp, S.J.; Flack, S.; Paximadas, D.; Spiess, K.; Adgey, C.; Griffiths, L.; Lim, R.; Trembling, P.; Williamson, K.; et al. The UK-PBC risk scores: Derivation and validation of a scoring system for long-term prediction of end-stage liver disease in primary biliary cholangitis. Hepatology 2016, 63, 930-950. [CrossRef] [PubMed]

9. Momah, N.; Silveira, M.G.; Jorgensen, R.; Sinakos, E.; Lindor, K.D. Optimizing biochemical markers as endpoints for clinical trials in primary biliary cirrhosis. Liver Int. 2012, 32, 790-795. [CrossRef]

10. European Association for the Study of the Liver. EASL Clinical Practice Guidelines: The diagnosis and management of patients with primary biliary cholangitis. J. Hepatol. 2017, 67, 145-172. [CrossRef] 
11. Nevens, F.; Andreone, P.; Mazzella, G.; Strasser, S.I.; Bowlus, C.; Invernizzi, P.; Drenth, J.P.; Pockros, P.J.; Regula, J.; Beuers, U.; et al. A Placebo-Controlled Trial of Obeticholic Acid in Primary Biliary Cholangitis. N. Engl. J. Med. 2016, 375, 631-643. [CrossRef]

12. Roberts, S.B.; Ismail, M.; Kanagalingam, G.; Mason, A.L.; Swain, M.G.; Vincent, C.; Yoshida, E.M.; Tsien, C.; Flemming, J.A.; Janssen, H.L.; et al. Real-World Effectiveness of Obeticholic Acid in Patients with Primary Biliary Cholangitis. Hepatol. Commun. 2020, 4, 1332-1345. [CrossRef]

13. Gomez, E.; Buey, L.G.; Molina, E.; Casado, M.; Conde, I.; Berenguer, M.; Jorquera, F.; Simón, M.-A.; Olveira, A.; Hernández-Guerra, M.; et al. Effectiveness and safety of obeticholic acid in a Southern European multicenter cohort of patients with primary biliary cholangitis and suboptimal response to ursodeoxycholic acid. Aliment. Pharmacol. Ther. 2020, 53, 519-530. [PubMed]

14. Hohenester, S.; Oude-Elferink, R.P.; Beuers, U. Primary biliary cirrhosis. Semin. Immunopathol. 2009, 31, 283-307. [CrossRef] [PubMed]

15. Prati, D.; Taioli, E.; Zanella, A.; Della Torre, E.; Butelli, S.; Del Vecchio, E.; Vianello, L.; Zanuso, F.; Mozzi, F.; Milani, S.; et al. Updated Definitions of Healthy Ranges for Serum Alanine Aminotransferase Levels. Ann. Intern. Med. 2002, 137, 1-10. [CrossRef]

16. Giannini, E.G.; Testa, R.; Savarino, V. Liver enzyme alteration: A guide for clinicians. CMAJ 2005, 172, 367-379. [CrossRef] [PubMed]

17. Classificazione del Medicinale per Uso Umano «Ocaliva». Available online: https://www.gazzettaufficiale.it/atto/serie_ generale / caricaDettaglioAtto / originario?atto.dataPubblicazioneGazzetta=2017-08-23\&atto.codiceRedazionale=17A05919 \&elenco30giorni=false (accessed on 3 November 2021).

18. Ludwig, J.; Dickson, E.R.; McDonald, G.S. Staging of chronic nonsuppurative destructive cholangitis (syndrome of primary biliary cirrhosis). Virchows Arch. A Pathol. Anat. Histol. 1978, 379, 103-112. [CrossRef]

19. De Franchis, R.; Baveno VI Faculty. Expanding consensus in portal hypertension: Report of the Baveno VI Consensus Workshop: Stratifying risk and individualizing care for portal hypertension. J. Hepatol. 2015, 63, 743-752. [CrossRef] [PubMed]

20. Bolondi, L.; Cillo, U.; Colombo, M.; Craxi, A.; Farinati, F.; Giannini, E.G.; Golfieri, R.; Levrero, M.; Pinna, A.D.; Piscaglia, F.; et al. Position paper of the Italian Association for the Study of the Liver (AISF): The multidisciplinary clinical approach to hepatocellular carcinoma. Dig. Liver Dis. 2013, 45, 712-723. [CrossRef] [PubMed]

21. Cazzagon, N.; Floreani, A. Primary biliary cholangitis: Treatment. Curr. Opin. Gastroenterol. 2021, 37, 99-104. [CrossRef] [PubMed]

22. Harms, M.H.; van Buuren, H.R.; Corpechot, C.; Thorburn, D.; Janssen, H.L.; Lindor, K.D.; Hirschfield, G.M.; Parés, A.; Floreani, A.; Mayo, M.J.; et al. Ursodeoxycholic acid therapy and liver transplant-free survival in patients with primary biliary cholangitis. J. Hepatol. 2019, 71, 357-365. [CrossRef] [PubMed]

23. D'Amato, D.; De Vincentis, A.; Malinverno, F.; Viganò, M.; Alvaro, D.; Pompili, M.; Picciotto, A.; Palitti, V.P.; Russello, M.; Storato, S.; et al. Real-world experience with obeticholic acid in patients with primary biliary cholangitis. JHEP Rep. 2021, 3, 100248. [CrossRef] [PubMed]

24. Gao, Y.; Li, L.; Li, B.; Zhan, Y. Response Rate and Impact on Lipid Profiles of Obeticholic Acid Treatment for Patients with Primary Biliary Cholangitis: A Meta-Analysis. Can. J. Gastroenterol. Hepatol. 2021, 2021, 8829510. [CrossRef] [PubMed]

25. Harms, M.H.; Hirschfield, G.M.; Floreani, A.; Mayo, M.J.; Parés, A.; Liberman, A.; Malecha, E.S.; Pencek, R.; MacConell, L.; Hansen, B.E. Obeticholic acid is associated with improvements in AST-to-platelet ratio index and GLOBE score in patients with primary biliary cholangitis. JHEP Rep. 2021, 3, 100191. [CrossRef]

26. FDA adds Boxed Warning to Highlight Correct Dosing of Ocaliva (Obeticholic Acid) for Patients with a Rare Chronic Liver Disease. Available online: https://www.fda.gov/drugs/drug-safety-and-availability/fda-adds-boxed-warning-highlightcorrect-dosing-ocaliva-obeticholic-acid-patients-rare-chronic-liver (accessed on 3 November 2021).

27. John, B.V.; Schwartz, K.; Levy, C.; Dahman, B.; Deng, Y.; Martin, P.; Taddei, T.H.; Kaplan, D.E. Impact of Obeticholic acid Exposure on Decompensation and Mortality in Primary Biliary Cholangitis and Cirrhosis. Hepatol. Commun. 2021, 5, 1426-1436. [CrossRef] 